\title{
PI3K/Akt signaling disruption affects autophagy in dystrophin-deficient myoblasts
}

Muhammad Dain Yazid

Hung-Chih Chen

\section{Video Byte}

Keywords: myoblasts, dystrophin, PI3K, Akt, PI3K/Akt signaling, autophagy, Duchenne muscular dystrophy, DMD, muscular dystrophy, PTEN, rictor-mTORC2, Fox03, autophagosome, LC3B-I/II, LCB3 lipidation, autophagic flux, mdx mouse, dfd13 myoblasts, Cell Communication and Signaling

Posted Date: March 1st, 2022

DOI: https://doi.org/10.21203/rs.3.rs-1405894/v1

License: (c) (i) This work is licensed under a Creative Commons Attribution 4.0 International License.

Read Full License 


\section{Abstract}

In individuals with Duchenne muscular dystrophy (DMD), lack of the protein dystrophin impairs autophagy and drastically disrupts myotube development. The PI3K/Akt/mTOR pathway is a vital autophagy-regulating pathway that also participates in skeletal muscle differentiation. However, the precise disruption of this pathway in the context of DMD remains unclear. To clarify the mechanism, researchers recently performed protein profiling on dystrophin-deficient myoblasts. The deficient (dfd13) myoblasts were not able to achieve terminal differentiation. They also exhibited strongly increased PTEN expression and perturbed $\mathrm{PI} 3 \mathrm{~K} / \mathrm{Akt} / \mathrm{mTOR}$ regulation. In addition, rictor-mTORC2 was inactivated, which caused FoxO3 misregulation and ultimately increased autophagy-related gene activation.

Autophagosome formation was excessive in the dystrophin-deficient myoblasts; however, subsequent LC3B-I to LC3B-II conversion and autophagic flux were decreased, consistent with the known autophagy disruption in DMD. Although further mechanistic research is needed, the results show that PTEN-PI3K/Akt pathway perturbation dysregulates autophagy in dystrophin-deficient myoblasts and suggest that manipulation of this pathway could have therapeutic effects in patients with DMD. 